\title{
Stochastic subgrid modelling for atmospheric large eddy simulations
}

\author{
Jorgen S. Frederiksen ${ }^{1} \quad$ Meelis J. Zidikheri $^{2}$
}

(Received 22 July 2008; revised 19 November 2008)

\begin{abstract}
Dynamical subgrid scale parameterizations of stochastic backscatter and eddy dissipation have been calculated for typical atmospheric turbulent flows on the sphere. A methodology based on a stochastic model representation of the subgrid scale eddies in direct numerical simulations, and with wide applicability to fluid flows, has been employed. Large eddy simulations incorporating these subgrid scale parameterizations are found to have energy spectra that compare closely with the results of higher resolution direct numerical simulations for both barotropic and baroclinic turbulent flows.
\end{abstract}

\section{Contents}

\section{Introduction}

http://anziamj . austms.org. au/ojs/index.php/ANZIAMJ/article/view/1365 gives this article, (c) Austral. Mathematical Soc. 2008. Published December 4, 2008. ISSN 1446-8735. (Print two pages per sheet of paper.) 
2 Quasigeostrophic models

3 Subgrid scale parameterizations

C494

4 Barotropic model results

C495

5 Baroclinic model results

C498

6 Conclusions

C501

References

C503

\section{Introduction}

In recent years there has increased interest in developing and employing improved dynamical subgrid scale parameterizations for atmospheric climate and weather prediction models (Frederiksen et al. [4]). In particular it has been demonstrated that some of the problems in climate simulations such as resolution dependence of the kinetic energy spectra may be cured (Frederiksen and Davies [2]) or ameliorated [4] by self-consistently determined dynamical subgrid scale parameterizations. It has also been realized that subgrid scale eddies not only drain enstrophy from the retained scales through an effective eddy viscosity but also inject enstrophy to the retained scales through a stochastic backscatter term [2]. In this study we apply a direct stochastic modelling approach to subgrid scale parameterizations (Frederiksen and Kepert [3]) that has been shown to have similar performance to parameterizations based on renormalized statistical closure theory ([2]; Frederiksen [1]; O'Kane and Frederiksen [5]).

In Section 2 we describe the quasigeostrophic models for atmospheric flows and turbulence in spherical geometry used in these studies and summarize the spectral equations for the spherical harmonic coefficients. Section 3 
presents a stochastic methodology, with wide applicability, for deriving subgrid scale parameterizations of eddy dissipation and stochastic backscatter from direct numerical simulations (DNS). In Sections 4 and 5 we compare large eddy simulations (LES) incorporating the subgrid scale parameterizations with higher resolution DNS for barotropic and baroclinic turbulent flows. Our conclusions are summarized in Section 6.

\section{Quasigeostrophic models}

For our studies of stochastic subgrid scale modelling of atmospheric large eddy simulations we consider barotropic and baroclinic flows and turbulence on the sphere described by quasigeostrophic equations. The quasigeostrophic two level model for baroclinic dynamics is discretized by expanding each of the fields in spherical harmonics with zonal wavenumber $m$ and total wavenumber $\mathrm{n}$. The spectral form of the two level equations are

$$
\frac{\partial q_{m n}^{j}}{\partial t}=i \sum_{p q} \sum_{r s} A_{n q s}^{m p r} \zeta_{-p q}^{j} q_{-r s}^{j}-D_{o}^{j}(m, n) \zeta_{m n}^{j}+\kappa\left(\tilde{q}_{m n}^{j}-q_{m n}^{j}\right)+f_{0}^{j}(m, n),
$$

where $\boldsymbol{j}=1$ is the upper level (typically $250 \mathrm{hPa}$ ) and $\boldsymbol{j}=2$ is the lower level (typically $750 \mathrm{hPa}$ ). Here the (reduced) potential vorticity spectral coefficients are defined by $q_{m n}^{j}=\zeta_{m n}^{j}-(-1)^{j}\left(\zeta_{m n}^{1}-\zeta_{m n}^{2}\right) F_{L}[n(n+1)]^{-1}$ where $\zeta_{m n}^{j}$ are the vorticity spectral coefficients; $f_{0}^{j}(m, n)$ are forcing terms. Also, $D_{0}^{j}(m, n)=\alpha^{j}+v_{0}^{j}[n(n+1)]^{\rho}+i \omega_{m n}^{j}$ is a generalized complex operator whose real part describes dissipation and imaginary part describes the frequency $\omega_{m n}^{j}=-\operatorname{Bm}[n(n+1)]^{-1}$ of Rossby waves; $\rho$ is a positive integer discussed below. These equations are nondimensional; we use the earth's radius as a length scale and the inverse of the earth's angular velocity as a time scale. The beta effect $B$, due to differential rotation, takes the value two with current scalings, but we also consider the case $B=0$. The layer coupling parameter $F_{L}$ is inversely proportional to the potential temperature 


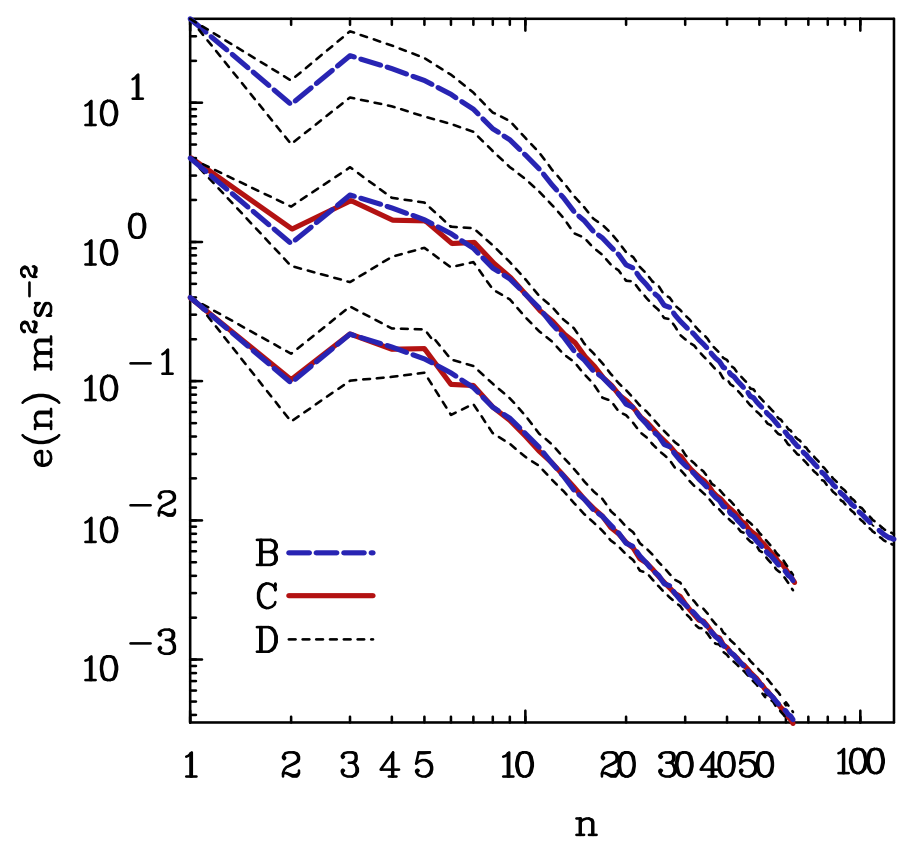

Figure 1: Kinetic energy spectra $e(n)\left(\mathrm{m}^{2} \mathrm{~s}^{-2}\right)$ as functions of total wavenumber $\mathrm{n}$ for (B) DNS at T126 (upper results), (C) LES at T63 with renormalized dissipation and renormalized noise forcing (middle results $\times 10^{-1}$ ) and LES at T63 with renormalized net dissipation (lower results $\left.\times 10^{-2}\right)$, and $(\mathrm{D}) e(\mathrm{n}) \pm$ standard deviation. 
difference between the levels. We also allow for a simple representation of the effects of differential heating through a specification of $\tilde{q}^{j}{ }_{m n}$. This is the value towards which $\mathbf{q}_{m n}^{j}$ is relaxed on a timescale given by $\kappa^{-1}$. The interaction coefficients $A_{\mathrm{nqs}}^{\mathrm{mpr}}=-\mathrm{K}_{\mathrm{nqs}}^{\mathrm{mpr}}[\mathbf{q}(\mathbf{q}+1)]^{-1}$, with $\mathrm{K}_{\mathrm{nqs}}^{\mathrm{mpr}}$ explicitly given by Frederiksen and Kepert [3], describe the non-linear coupling between the streamfunction and potential vorticity. The barotropic model may be obtained from the baroclinic equations by setting $F_{L}=0$.

\section{$3 \quad$ Subgrid scale parameterizations}

Let $\mathbf{q}$ denote the column vector of spectral coefficients $\mathbf{q}_{m n}^{j}$. Then on reducing the resolution from $T$ (for DNS) to $R$ (for LES) we write the tendency as $\mathbf{q}_{\mathbf{t}}=\left(\mathbf{q}_{\mathbf{t}}\right)_{R}+\left(\mathbf{q}_{\mathbf{t}}\right)_{S}$ where $\mathbf{q}_{\mathbf{t}}=\partial \mathbf{q}(\mathbf{t}) / \partial t$. The vector $\left(\mathbf{q}_{\mathbf{t}}\right)_{R}$ represents the resolved scale tendency and $\left(\mathbf{q}_{\mathrm{t}}\right)_{\mathrm{S}}$ represents the subgrid scale tendency. Now, $\mathbf{q}$ consists of a transient part $\hat{\mathbf{q}}=\mathbf{q}-\overline{\mathbf{q}}$ and a mean part $\overline{\mathbf{q}}$. Similarly, the subgrid tendency has both mean and transient parts $\left(\mathbf{q}_{\mathbf{t}}\right)_{S}=\mathbf{f}_{\mathbf{m}}+\left(\hat{\mathbf{q}}_{\mathbf{t}}\right)_{S}$, where $\mathbf{f}_{\mathbf{m}}$ and $\left(\hat{\mathbf{q}}_{\mathrm{t}}\right)_{S}$ are the mean and transient parts, respectively. In this study, the mean part is simply calculated from a high resolution simulation as the time average of the subgrid tendency.

The subgrid terms are represented by the stochastic equation

$$
\left(\frac{\partial \hat{\mathbf{q}}(\mathrm{t})}{\partial \mathrm{t}}\right)_{\mathrm{S}}=-\mathbf{D}_{\mathrm{d}} \hat{\mathbf{q}}(\mathrm{t})+\hat{\mathbf{f}}_{\mathrm{b}}(\mathrm{t})
$$

Here, $\mathbf{D}_{\mathbf{d}}$ is defined as the drain dissipation matrix and $\hat{\mathbf{f}}_{\mathbf{b}}$ is a random forcing vector. Frederiksen and Kepert [3] formulated the stochastic model for the drain dissipation matrix to account for memory effects of turbulent eddies by analogy with the direct interaction closure approach of Frederiksen and Davies [2]. Thus, the drain dissipation matrix has the integral form

$$
D_{d}=-\left[\int_{t_{0}}^{t} d s\left\langle\left(\frac{\partial \hat{\mathbf{q}}(s)}{\partial t}\right)_{s} \hat{\mathbf{q}}^{\dagger}\left(t_{0}\right)\right\rangle\right]\left[\int_{t_{0}}^{t} \mathrm{ds}\left\langle\hat{\mathbf{q}}(t) \hat{\mathbf{q}}^{\dagger}\left(t_{0}\right)\right\rangle\right]^{-1} .
$$


Here, angle brackets denote either ensemble or time averaging; in this study, we consider the latter; also $\dagger$ denotes Hermitian conjugate.

The covariance matrix for the subgrid nonlinear noise $\mathbf{F}_{\mathbf{b}}=\mathrm{F}_{\mathbf{b}}+\mathrm{F}_{\mathbf{b}}^{\dagger}$ where $F_{\mathbf{b}}=\left\langle\hat{\mathbf{f}}_{\mathbf{b}}(\mathbf{t}) \hat{\mathbf{q}}^{\dagger}(\mathrm{t})\right\rangle$ may be obtained from the Lyapunov or balance equation

$$
\begin{aligned}
& \left\langle\left(\frac{\partial \hat{\mathbf{q}}(t)}{\partial t}\right)_{S} \hat{\mathbf{q}}^{\dagger}(t)\right\rangle+\left\langle\hat{\mathbf{q}}(t)\left(\frac{\partial \hat{\mathbf{q}}(t)}{\partial t}\right)_{S}^{\dagger}\right\rangle \\
& =-\mathbf{D}_{\mathbf{d}}\left\langle\hat{\mathbf{q}}(t) \hat{\mathbf{q}}^{\dagger}(t)\right\rangle-\left\langle\hat{\mathbf{q}}(t) \hat{\mathbf{q}}^{\dagger}(t)\right\rangle \mathbf{D}_{\mathbf{d}}^{\dagger}+\mathbf{F}_{\mathbf{b}}(t)
\end{aligned}
$$

after computing $\mathbf{D}_{\mathbf{d}}$.

In general the nonlinear noise may be coloured but we find that once it has been calculated as described above it is sufficient to model it by white noise in the subsequent LES. Thus, the forcing $\hat{\mathbf{f}}_{\mathbf{b}}(\mathrm{t})$ takes the white noise form $\left\langle\hat{\mathbf{f}}_{\mathbf{b}}(t) \hat{\mathbf{f}}_{\mathbf{b}}^{\dagger}\left(\mathbf{t}^{\prime}\right)\right\rangle=\mathbf{F}_{\mathbf{b}}(\mathbf{t}) \delta\left(t-t^{\prime}\right)$, where $\delta\left(t-t^{\prime}\right)$ is the Dirac delta function.

We also consider a deterministic parameterization where $\mathbf{F}_{\mathbf{b}}=\mathrm{F}_{\mathbf{b}}+\mathrm{F}_{\mathbf{b}}^{\dagger}$ is represented by a backscatter dissipation matrix defined by

$$
\mathbf{D}_{\mathbf{b}}=-\mathrm{F}_{\mathbf{b}}\left[\left\langle\hat{\mathbf{q}}(\mathrm{t}) \hat{\mathbf{q}}^{\dagger}(\mathrm{t})\right\rangle\right]^{-1} .
$$

Then we define the net dissipation matrix by

$$
\mathbf{D}_{\mathrm{n}}=\mathbf{D}_{\mathrm{d}}+\mathbf{D}_{\mathrm{b}}
$$

\section{Barotropic model results}

In this section we consider first the case of barotropic and isotropic turbulence with no mean field, mean forcing or rotation $(B=0)$ and then go on to more complex flows in Section 5. We simulate a typical atmospheric isotropic spectrum at the resolution of triangular truncation T126 


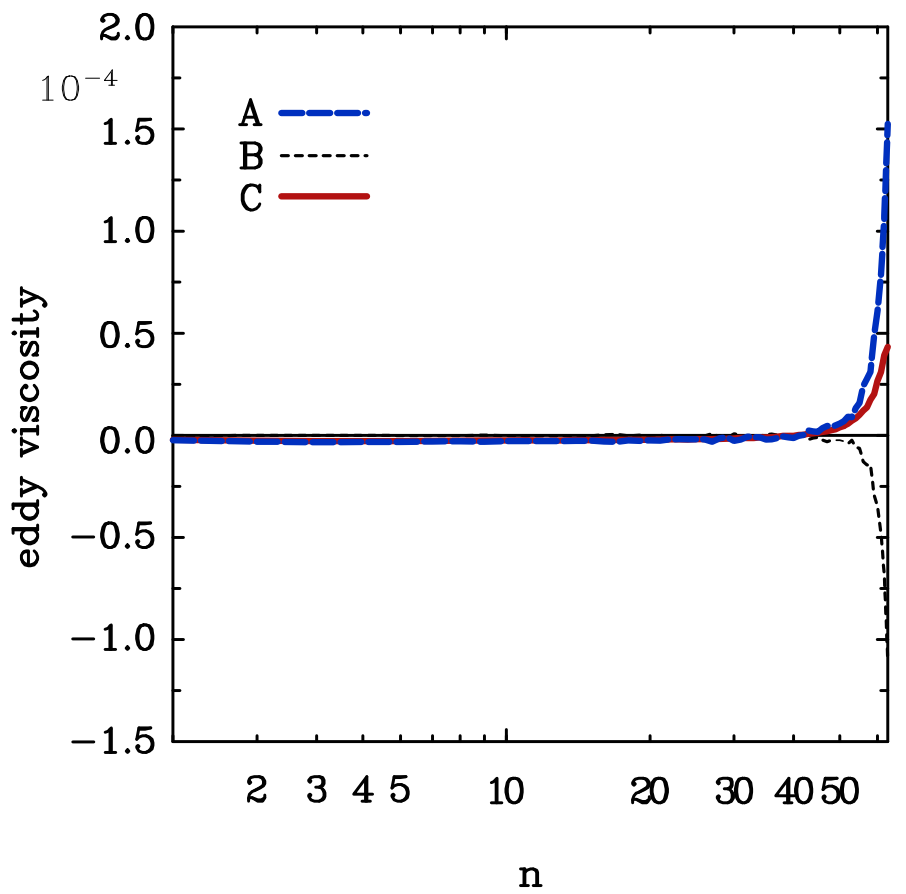

Figure 2: Nondimensional subgrid scale parameterizations of (A) eddy drain viscosity $v_{d}(n)$, (B) eddy backscatter viscosity $v_{b}(n)$, and (C) net eddy viscosity $v_{n}(n)$ as functions of $n$. 
which retains 126 wavenumbers and corresponds to $384 \times 192$ grid points (in longitude $\times$ latitude). The flow is maintained by random forcing $\hat{f}_{0}$ with variance $F_{0}$ (not shown) and typical bare dissipation $D_{0}(m, n)=1.014 \times 10^{-2}$ for $2 \leq n \leq 21$ and $D_{0}(m, n)=1.014 \times 10^{-2}+2.115 \times 10^{-5} n(n+1)$ for $22 \leq \mathrm{n} \leq 126$ in non-dimensional units. Here, the drag contribution $\left(1.014 \times 10^{-2}\right)$ corresponds to an e-folding time of 15.6 days, and the Laplacian $(\rho=1)$ contribution $\left(2.115 \times 10^{-5}\right)$ corresponds to $6.25 \times 10^{-4} \mathrm{~m}^{2} \mathrm{~s}^{-1}$ in dimensional units.

Figure 1 (upper results) show the kinetic energy $e(n)$, averaged over $m$ and averaged over the last 100 days of a 150 day integration with the barotropic vorticity equation, and the standard deviations about the means. We regard the T126 DNS spectra as the benchmarks against which we compare the LES at lower resolution, chosen as T63 (192 ×96 grid points), with dynamical subgrid scale parameterizations. These subgrid scale parameterizations are based on truncating the T126 results back to T63 and are calculated as described in Section 3. Because there is only one level and because the barotropic turbulence is isotropic, the matrices $\mathbf{D}$ and $\mathbf{F}_{\mathbf{b}}$ of Section 2 become scalars $D$ and $F_{b}$ that depend only on the total wavenumber $n$. Figure 2 shows the viscosities $v_{d}=D_{d}[n(n+1)]^{-1}, v_{b}=D_{b}[n(n+1)]^{-1}$ and $v_{n}=D_{n}[n(n+1)]^{-1}=v_{d}+v_{b}$ which depend only on the total wavenumber $n$. These viscosities, and the corresponding dissipations have a cusp behaviour at the smallest scales. We also note that the net viscosity $v_{n}$ is the relatively small difference between drain due to the drain viscosity $v_{d}$ and injection due to the negative backscatter viscosity $v_{\mathrm{b}}$.

Large eddy simulations have been performed at T63 with the renormalized drain dissipation $D_{r}=D_{d}+D_{0}$ and white noise renormalized forcing $\hat{f}_{r}=$ $\hat{f}_{b}+\hat{f}_{0}$ and also with the renormalized net dissipation $D_{r n}=D_{n}+D_{0}$ and bare forcing $\hat{f}_{0}$. Figure 1 (middle results) show the LES kinetic energy spectra $e(n)$, averaged over the last 100 days of a 150 day simulation with $D_{r}$ and $\hat{f}_{r}$, as well as the standard deviations about these spectra. Also shown are the energy spectra of the DNS truncated back to T63. The lower results in Figure 1 
(a)

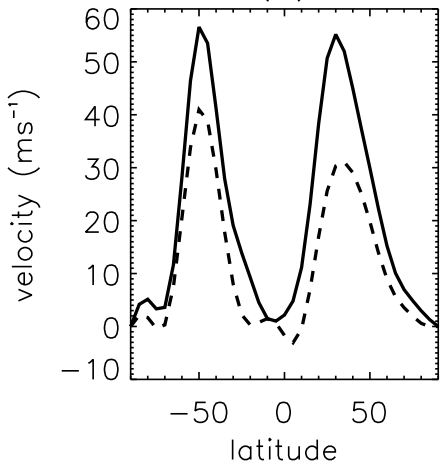

(b)

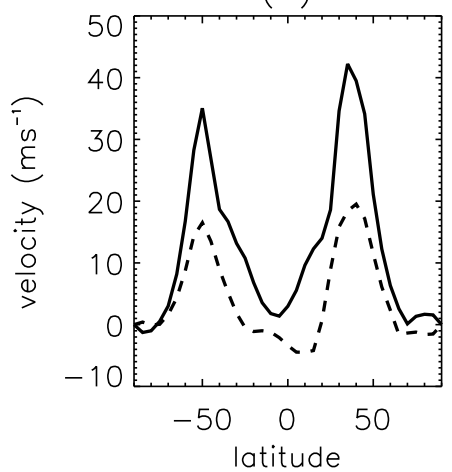

FigURE 3: Longitudinally averaged (a) relaxation winds, and (b) time averaged actual winds $\left(\mathrm{ms}^{-1}\right)$ for Level 1 (solid) and Level 2 (dashed).

are the corresponding findings with $D_{\mathrm{rn}}$ and $\hat{f}_{\mathrm{o}}$. Both sets of LES results in Figure 1 are in very good agreement with DNS truncated back to T63.

\section{$5 \quad$ Baroclinic model results}

Next we consider more realistic atmospheric flows with differential rotation $(\mathrm{B}=2)$ and large scale mean jets in the baroclinic model.

The following parameters have been chosen for DNS, again at T126, with the baroclinic model to reproduce typical atmospheric spectra. The drag, $\alpha^{j}$, has been set to a damping time of 20 days for Level 1 and 5 days for Level 2; the bare hyperviscosity $v_{0}^{j}=3.9 \times 10^{32} \mathrm{~m}^{8} \mathrm{~s}^{-1}$ for both layers; the order of the Laplacian operator $\rho=4$. The potential vorticity relaxation, $\tilde{\mathrm{q}}_{j}$, has been chosen to correspond to vertically sheared mid-latitude jets in both hemispheres. The latitudinal profiles of the corresponding zonal wind jets $\tilde{u}^{j}$ 
are shown in Figure 3(a); the relaxation time, $\mathbf{k}^{-1}$, is 11.6 days. The layer coupling constant, $\mathrm{F}_{\mathrm{L}}=100$ in non-dimensional units or $2.5 \times 10^{-12} \mathrm{~m}^{-2}$ in dimensional units, corresponds to a deformation radius of about $500 \mathrm{~km}$.

The model has been integrated to statistical steady state and then further stepped forward in time for 208 days. Figure 3(b) shows the zonally and time averaged zonal wind at statistical steady state. The mean flow is dominated by the mid-latitude jet streams in both hemispheres; the jet streams are sheared in the vertical. This shear creates baroclinic instability spectrally peaked in the vicinity of wavenumber 10. Figure 4 shows the Level 1 kinetic energy, $e(n)$, of the DNS truncated back to T63; at smaller scales it follows an $n^{-3}$ power law (not shown) as in Figure 1 for the barotropic case.

Again, we use the stochastic modelling approach to calculate the dynamical subgrid scale parameterizations for LES at T63. We find that successful large eddy simulations result from parameterizations that are inhomogeneous in the vertical, as expected from the vertically sheared relaxation winds in Figure 3, and homogeneous in the horizontal. This is consistent with the quasi-diagonal turbulence closure theory [1] and its ability to simulate the statistics of DNS in the presence of mean flow inhomogeneities [5]. The eddy dissipation and nonlinear noise terms then take the forms $D^{j k}(m, n)$ and $\mathrm{F}_{\mathrm{b}}^{\mathrm{jk}}(\mathrm{m}, \mathrm{n})$, where $j, k=1,2$; that is, they reduce to $2 \times 2$ matrices at each wavevector $(\mathfrak{m}, \mathfrak{n})$. As for the barotropic problem of Section 4 , the parameterizations are essentially isotropic at the smallest scales where they have a cusp. Near the cusp, where the contributions to the parameterizations are largest, the term $n(n+1)$ is much larger than $F_{L}$, and, since the potential vorticity $\mathrm{q}_{m n}^{j}=\zeta_{m n}^{j}-(-1)^{j}\left(\zeta_{m n}^{1}-\zeta_{m n}^{2}\right) F_{L}[n(n+1)]^{-1}$, the two levels become uncoupled. This results in the cross terms in the parameterizations being much smaller than the diagonal components at T63. For these reasons we only display the diagonal and isotropized components of the parameterizations in this paper.

The subgrid scale parameterizations $\mathrm{D}_{\mathfrak{n}}^{\mathrm{jj}}, \mathrm{D}_{\mathrm{d}}^{\mathrm{jj}}$, and $\mathrm{F}_{\mathrm{b}}^{\mathrm{jj}}$ are shown in Figure 5. The dashed red lines in Figures 5(a) and 5(b) represent the net 


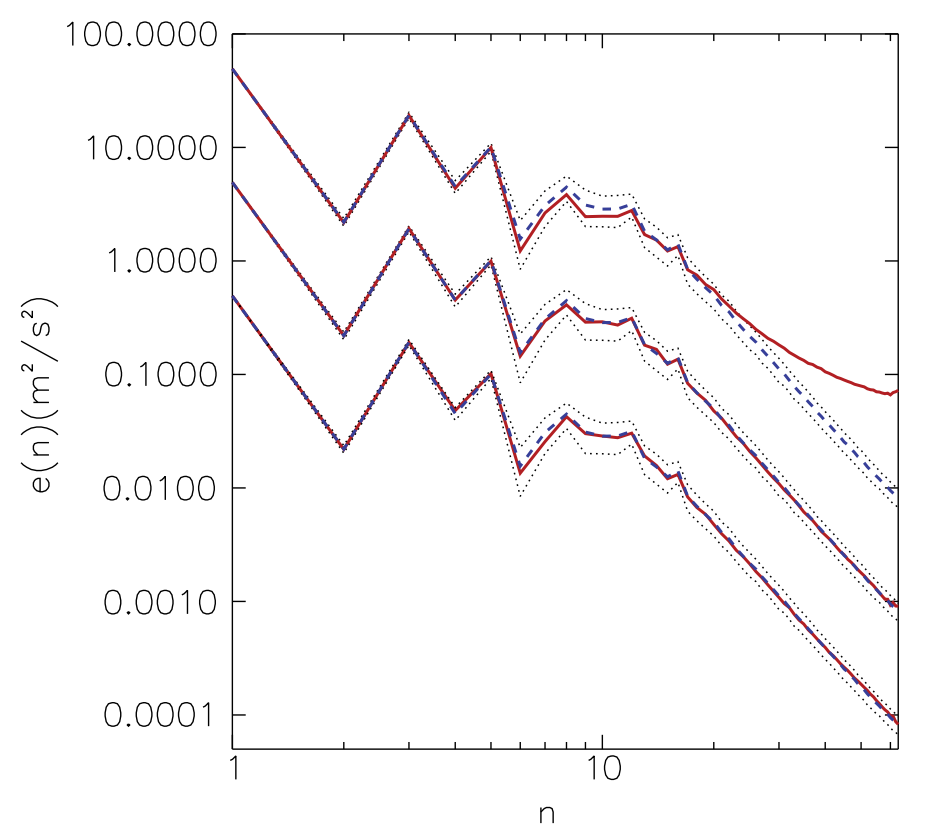

Figure 4: Kinetic energy spectra $e(n)\left(\mathrm{m}^{2} \mathrm{~s}^{-2}\right)$ at Level 1 as functions of total wavenumber $(n)$ for LES at T63 (solid red); for DNS at T126 truncated back to T63 (dashed blue); and DNs e( $\mathrm{n}) \pm$ standard deviation (dotted black). The top results are for LES with bare dissipation; the middle results $\left(\times 10^{-1}\right)$ are for LES with deterministic renormalized net dissipation; the bottom results $\left(\times 10^{-2}\right)$ are for LES with renormalized drain dissipation and stochastic backscatter. 
dissipation parameters $D_{n}$ and the solid blue lines the drain dissipations $D_{d}$. We find that the parameters $D_{n}$ are roughly of the same size across the two levels. The corresponding parameters $\mathrm{F}_{\mathrm{b}}$ are shown in Figures $5(\mathrm{c})$ and $5(\mathrm{~d})$. The forcing variance $F_{b}^{11}$ is over an order of magnitude greater than $F_{b}^{22}$. Note that the mean forcing, $\mathbf{f}_{\mathbf{m}}$, is insignificant in magnitude because the baroclinic injection is fully resolved at resolution T63.

We performed LES at T63 for three different specifications of the dissipation. Firstly, the same bare dissipation (and forcing) as used for the DNS has been used. Secondly, the renormalized net dissipation, which is the sum of the net eddy dissipation and the bare dissipation, has been employed. Finally, LES with renormalized drain dissipation, which is the sum of the eddy drain dissipation and the bare dissipation, and stochastic backscatter has been performed. The Level 1 kinetic energy spectra $e(n)$ of the three LES are depicted in Figure 4. The top curves show the LES with bare parameters; note that the tail of the LES kinetic energy spectrum lifts due to insufficient drain of potential enstrophy. The middle curves show the LES with the deterministic renormalized net dissipation and the bottom curves show the LES with renormalized drain dissipation and stochastic backscatter (parameters shown in Figure 5). Both parameterizations perform very well, with LES in excellent agreement with higher resolution DNS at T126. Very similar agreement is obtained for the Level 2 kinetic energy and for the potential energy (not shown).

\section{Conclusions}

We have implemented a stochastic method for deriving dynamical subgrid scale parameterizations of eddy dissipation and stochastic backscatter, needed for LES, from higher resolution DNS. For both barotropic and baroclinic turbulent flows, LES at triangular truncation T63, and with eddy drain dissipation and stochastic backscatter parameterizations, have been compared with 
(a)

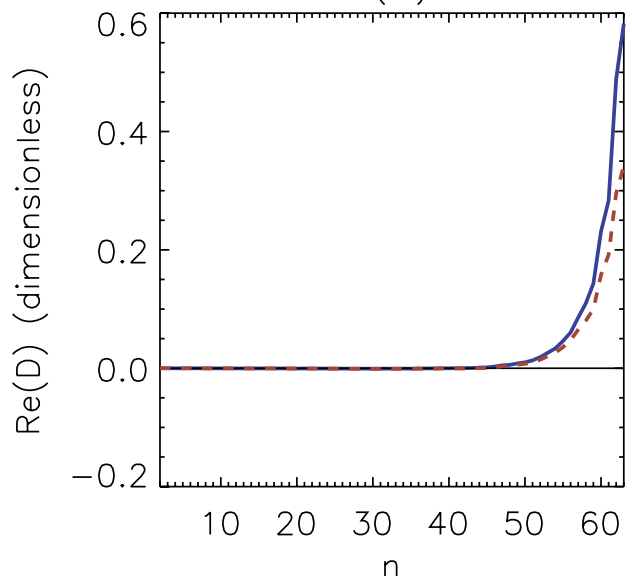

(c)

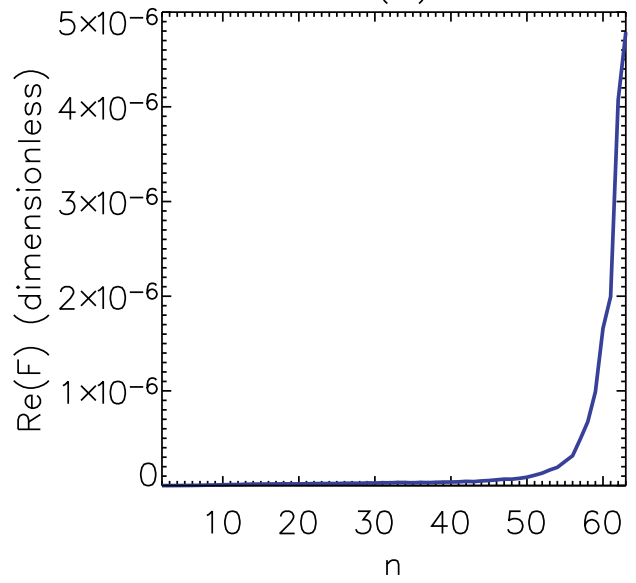

(b)

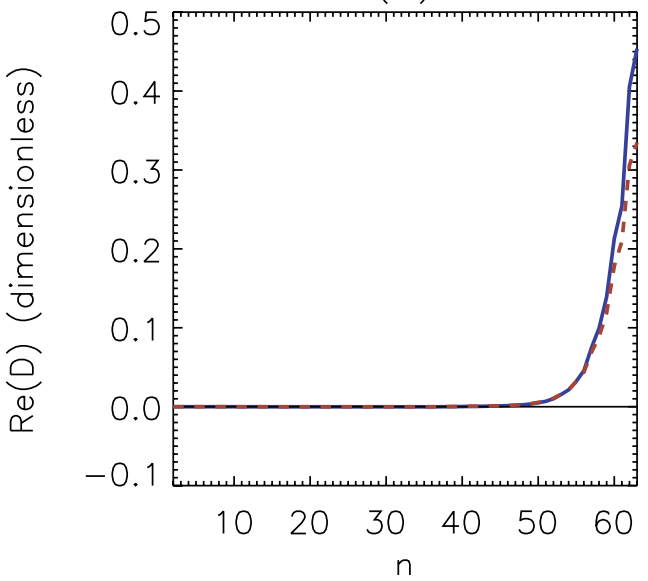

(d)

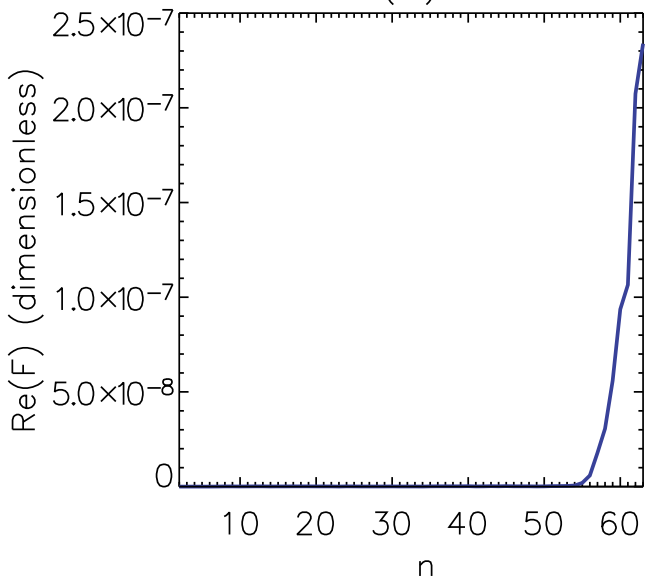

Figure 5: Eddy dissipation and stochastic backscatter coefficients (a) $\operatorname{Re}\left(\mathrm{D}^{11}\right)$, (b) $\operatorname{Re}\left(\mathrm{D}^{22}\right)$, (c) $\operatorname{Re}\left(\mathrm{F}_{\mathrm{b}}^{11}\right)$, and (d) $\operatorname{Re}\left(\mathrm{F}_{\mathrm{b}}^{22}\right)$ as functions of total wavenumber $\mathrm{n}$ at T63. Eddy drain dissipation $\operatorname{Re}\left(\mathrm{D}_{\mathrm{d}}^{\mathrm{jj}}\right)$ and stochastic backscatter $\operatorname{Re}\left(\mathrm{F}_{\mathrm{b}}^{\mathrm{jj}}\right)$ are shown in solid blue line and net eddy dissipation $\operatorname{Re}\left(\mathrm{D}_{\mathfrak{n}}^{\mathrm{jj}}\right)$ in dashed red. 
higher resolution DNS. As well, LES have been carried out with a deterministic net eddy dissipation. In our studies, with and without differential rotation and with and without large scale jets, we found that both formulations of LES perform very well in reproducing the spectra of DNS. This is in contrast to LES employing the original bare dissipation formulation, used for the DNS, for which the tail of the LES kinetic energy spectrum lifts due to insufficient dissipation at the lower resolution. The direct stochastic modelling method for subgrid scale parameterizations has the advantages of simplicity and wide applicability to turbulent fluid flows without the need for complex closures. It has similar performance to approaches based on closures including overcoming the long standing problems with ad hoc dissipation operators [2]. We plan in future to use the stochastic modelling technique to develop subgrid scale parameterizations for inhomogeneous turbulent flows in more complex atmospheric and oceanic models.

\section{References}

[1] Frederiksen, J.S., Subgrid-scale parameterizations of eddy-topographic force, eddy viscosity, and stochastic backscatter for flow over topography, J. Atmos. Sci. 56, 1999, 1481-1494. doi:10.1175/1520-0469(1999)056;1481:SSPOET¿2.0.CO;2 C491, C499

[2] Frederiksen, J.S., and A.G. Davies, Eddy viscosity and stochastic backscatter parameterizations on the sphere for atmospheric circulation models, J. Atmos. Sci., 54, 1997, 2475-2492. doi:10.1175/1520-0469(1997)054¡2475:EVASBP ¿2.0.CO;2 C491, C494, C503

[3] Frederiksen, J.S. and S.M. Kepert, Dynamical subgrid-scale parameterizations from direct numerical simulations, J. Atmos. Sci., 63, 2006, 3006-3019. doi:10.1175/JAS3795.1 C491, C494 
[4] Frederiksen, J.S. and M.R. Dix and A.G. Davies, The effects of closure-based eddy diffusion on the climate and spectra of a GCM, Tellus A, 55, 2003, 31-44. doi:10.1034/j.1600-0870.2003.201329.x C491

[5] O'Kane, T.J. and J.S. Frederiksen, Statistical dynamical subgrid-scale parameterizations for geophysical flows, Physica Scripta, 78, 2008, in press. C491, C499

\section{Author addresses}

1. Jorgen S. Frederiksen, CSIRO Marine and Atmospheric Research, Aspendale, Victoria, Australia.

mailto: jorgen.frederiksen@csiro.au

2. Meelis J. Zidikheri, Department of Theoretical Physics, Research School of Physical Sciences and Engineering, Australian National University, Canberra, Australia. 